\title{
A Green Slab Waveguide for Plasmonic Sensors Based on Bacterial Cellulose ${ }^{\dagger}$
}

\author{
Nunzio Cennamo ${ }^{1}$, Carlo Trigona ${ }^{2, *}$, Salvatore Graziani ${ }^{2}$, Luigi Zeni ${ }^{1}$, Francesco Arcadio ${ }^{1}$, \\ Giovanna Di Pasquale ${ }^{3}$ and Antonino Pollicino ${ }^{4}$ \\ 1 Dept. of Engineering, University of Campania Luigi Vanvitelli, Via Roma 29, 81031 Aversa, Italy; \\ nunzio.cennamo@unicampania.it (N.C.); luigi.zeni@unicampania.it (L.Z.); \\ francesco.arcadio@unicampania.it (F.A.) \\ 2 D.I.E.E.I. University of Catania, Viale Andrea Doria 6, 95125 Catania, Italy; salvatore.graziani@dieei.unict.it \\ 3 D.S.C., Dipartimento di Scienze Chimiche University of Catania, Viale Andrea Doria 6, 95125 Catania, Italy; \\ giovanna.dipasquale@dii.unict.it \\ 4 D.I.C.A.R., University of Catania, Viale Andrea Doria 6, 95125 Catania, Italy; apollicino@unict.it \\ * Correspondence: carlo.trigona@dieei.unict.it \\ $+\quad$ Presented at the 7th International Symposium on Sensor Science, Napoli, Italy, 9-11 May 2019. \\ Published: 13 August 2019
}

\begin{abstract}
We use as optical waveguide a green composite, based on bacterial cellulose (BC). More specifically, we have sputtered a thin gold film on this innovative slab waveguide for obtaining a Localized Surface Plasmon Resonance (LSPR) sensor. Experimental results confirm the possibility of using the $\mathrm{BC}$ based composite as an environmental friendly optical sensor platform with plasmonic capabilities, which could be exploited for realizing disposable biosensors. The new optical sensor has been used by combining it with optical fibers. The fibers connect the green disposable optical sensor with a light source and with a spectrometer. The device has been tested by measuring the refractive index of different water-glycerin solutions.
\end{abstract}

Keywords: optical sensors; sustainable development; localized surface plasmon resonance; bacterial cellulose; refractive index measurement

\section{Introduction}

Surface plasmon resonance (SPR) and localized surface plasmon resonance (LSPR) bio-chemical sensors in optical fibers are suitable for on-site and real-time monitoring of different analytes. As such, they play an important role in many research fields [1-5]. Many solutions have been proposed in order to optimize the performances of the SPR/LSPR sensors in terms of throughputs, reliability, robustness and miniaturization [6-11]. The optical fiber sensors can be defined as intrinsic and extrinsic, according to the interaction of the fiber with the analyzed medium (intrinsic) or its use as a mere waveguide allowing the launch of the light to the sensing region and its collection (extrinsic). The sensing scheme may be founded on reflection, where the light source and the detector lay on the same side of the fiber, or on transmission, where they are on opposite sides.

In this work, we show a transmission extrinsic optical fiber sensor, based on a slab waveguide of bacterial cellulose (BC), covered by a gold film. Cellulose is the most ambulant organic polymer present in nature. It is biocompatible and fully biodegradable [12]. Eventually, it can be processed to be used in "non conventional" flexible and green electronics. Cellulose is, generally, obtained by pant sources by the pulp industry. Unfortunately, the extraction of cellulose requires relevant amounts of both energy and resources (fresh water). Eventually, waste products are obtained, which can cause pollution, if released in the environment [13]. Because of the production process, the 
appeal of cellulose as a candidate for realizing greener sensing systems is greatly reduced. Though BC shares with plant-derived cellulose the same chemical structure, it is obtained by totally different sources. BC is produced, as a matter of fact, by some bacteria, in suitable cultures [14]. The culturing of $\mathrm{BC}$ is possible in typical laboratory environmental conditions. Moreover, no relevant quantities of energy and water are required. Because of the described production procedure required for $B C$, it is considered a greener alternative to plant-derived cellulose. Not less important, BC is produced in a much purer form than cellulose derived from plant sources.

As a consequence of the considerations reported above, a growing interest has been devoted to $\mathrm{BC}$ as a base component for realizing green transducers. Recently, some of the authors, have shown the possibility of using BC for realizing mechano-electric transducers [15-16].

Here, the possibility of exploiting BC based compounds for realizing optical sensors is addressed. A plasmonic sensor exploiting a BC thin film, with embedded silver nanoparticles has already been presented by NahidPourreza et al. [17], but their approach (a BC nanopaper, with silver nanoparticles) differs from the one proposed in this paper mainly because the metal (gold in our case) covers the cellulose wires so configuring a kind of nanowires able to excite LSPR.

\section{Plasmonic Sensor System}

In this work, we have covered by a thin gold film $(60 \mathrm{~nm})$ a BC-based composite, similar to that already used as an accelerometer [16]. Figure 1a shows the SEM image of the used bacterial cellulose based compound.

The procedure for the fabrication of the plasmonic platform includes only one step: gold is sputtered on the top of the CB-based paper, by using a sputtering machine (Bal-Tec SCD 500). In particular, the sputtering was repeated three times with a current of $60 \mathrm{~mA}$ for $35 \mathrm{~s} \mathrm{(20} \mathrm{nm} \mathrm{per} \mathrm{step).}$ The green plasmonic sensor platform has been characterized using a simple experimental configuration. The setup is based on a halogen lamp (HL-2000-LL, Ocean Optics), an optical coupler (50:50), two optical fibers connected with two similar spectrometers (USB2000+UV-VIS spectrometer, Ocean Optics), and two green slab waveguides of bacterial cellulose, one covered by gold (sensor) and one without gold (reference). Figure $1 \mathrm{~b}$ shows the experimental setup.

The spectral emission of the lamp ranges from $360 \mathrm{~nm}$ to $1700 \mathrm{~nm}$ and the spectrometer is sensitive from $300 \mathrm{~nm}$ to $1050 \mathrm{~nm}$.

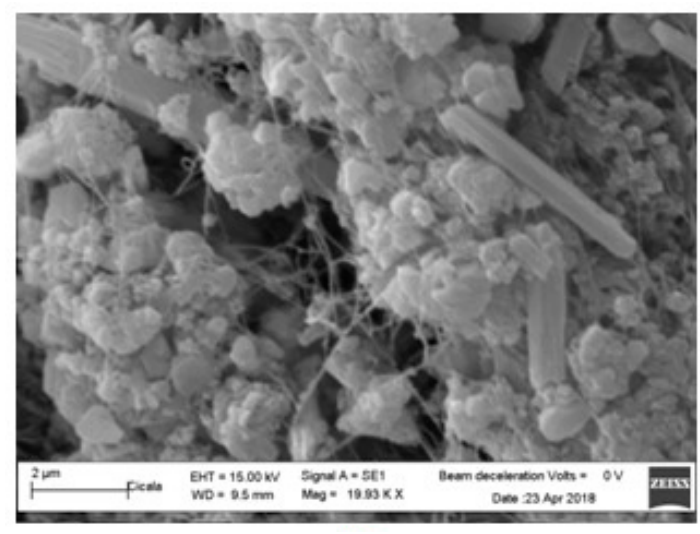

(a) 


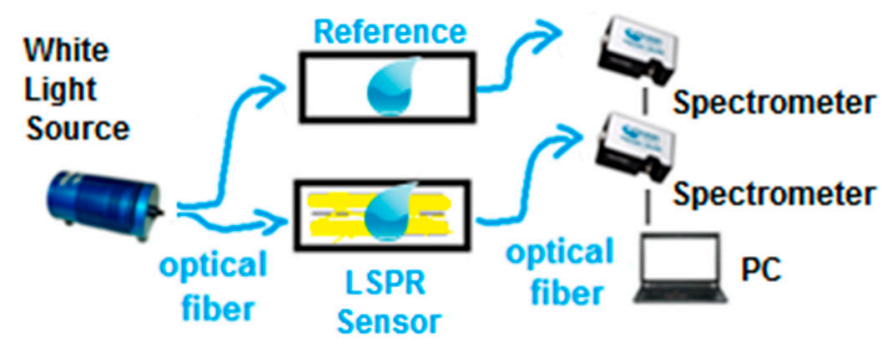

(b)

Figure 1. (a) SEM image of the bacterial cellulose. (b) The experimental setup used to test the plasmonic sensor.

The measurements have been performed by using different water-glycerin solutions in contact with the plasmonic slab waveguide. In Figure 2 are presented the experimentally obtained LSPR transmission spectra, normalized to the spectra achieved by the reference slab waveguide with the same surrounding medium, for three different water-glycerin solutions, with refractive index ranging from 1.332 to 1.350 .

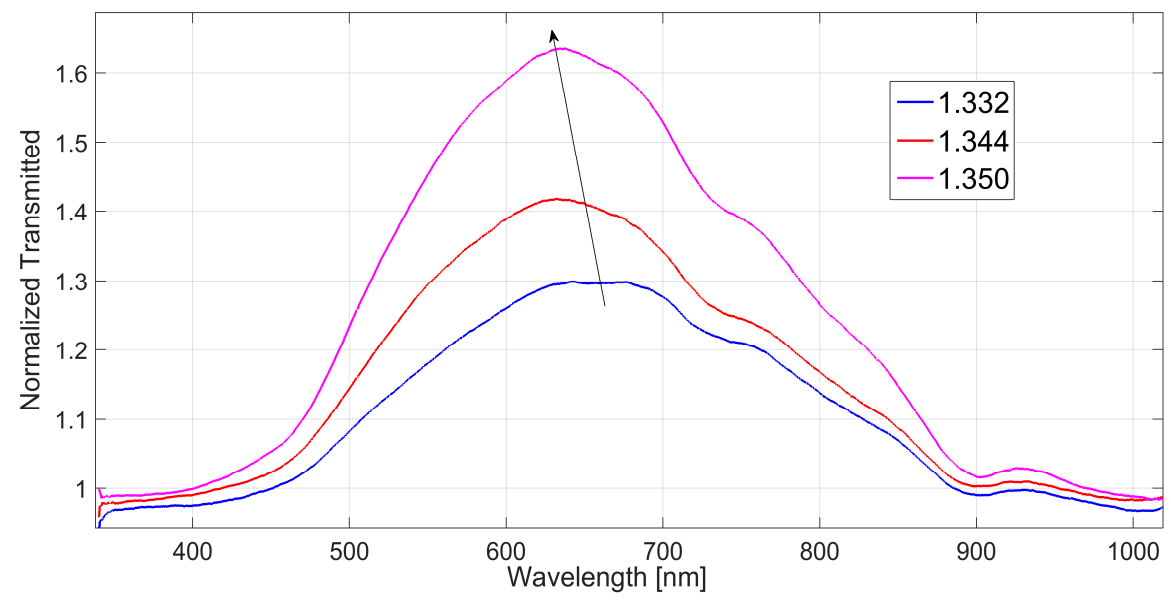

Figure 2. LSPR transmission spectra of the green plasmonic sensor obtained with three different water-glycerin solutions.

\section{Conclusions}

The proposed analysis shows that the chosen green slab waveguide, covered by gold, can be used to make environmental friendly LSPR sensor platforms, useful to realize disposable biosensors. Preliminary experimental results, obtained with different water-glycerin solutions, indicate that the sensor's response to refractive index changes is satisfactory.

\section{References}

1. Wang, X.D.; Wolfbeis, O.S. Fiber-Optic Chemical Sensors and Biosensors (2013-2015). Anal. Chem. 2016, 88, 203-227.

2. Anuj, K.; Sharma, R.J.; Gupta, B.D. Fiber-optic sensors based on surface Plasmon resonance: a comprehensive review. IEEE Sens. J. 2007, 7, 1118-1129.

3. Gupta, B.D.; Verma, R.K. Surface plasmon resonance-based fiber optic sensors: Principle, probe designs, and some applications. J. Sens. 2009, 2009, 1-12.

4. Trouillet, A.; Ronot-Trioli, C.; Veillas, C.; Gagnaire, H. Chemical sensing by surface plasmon resonance in a multimode optical fibre. Pure Appl. Opt. 1996, 5, 227. 
5. Leung, A.; Shankar, P.M.; Mutharasan, R. A review of fiber-optic biosensors. Sens Actuators B Chem. 2007, 125, 688-703.

6. Kanso, M.; Cuenot, S.; Louarn, G. Sensitivity of optical fiber sensor based on surface plasmon resonance: Modeling and experiments. Plasmonics 2008, 3, 49-57.

7. Dwivedi, Y.S.; Sharma A. K.; Gupta, B.D. Influence of design parameters on the performance of a surface plasmon sensor based fiber optic sensor. Plasmonics 2008, 3, 79-86.

8. Iga, M.; Seki, A.; Watanabe, K. Gold thickness dependence of SPR-based hetero-core structured optical fiber sensor. Sens. Actuators B: Chem.2005, 106, 363.

9. Sharma, A.K.; Gupta, B.D.;On the sensitivity and signal to noise ratio of a step-index fiber optic surface plasmon resonance sensor with bimetallic layers. Opt. Commun. 2005, 245, 159-169.

10. Jin, Y.; Granville, A.M. Polymer Fiber Optic Sensors-A Mini Review of their Synthesis and Applications. J. Biosens. Bioelectron. 2016, 7, 1-11.

11. Cennamo, N.; Pesavento, M.; De Maria, L.; Galatus, R.; Mattiello, F.; Zeni, L. Comparison of different photoresist buffer layers in SPR sensors based on D-shaped POF and gold film. In Proceedings of 25th International Conference on Optical Fiber Sensors, OFS 2017, Jeju, South Korea, 24-28 April 2017; pp. 1-4.

12. Kanaparthi, S.; Badhulika, S. Low cost, flexible and biodegradable touch sensor fabricated by solvent-free processing of graphite on cellulose paper. Sens. Actuators B: Chem. 2017, 242, 857-864.

13. Mangayil, R.; Rajala, S.; Pammo, A.; Sarlin, E.; Luo, J.; Santala, V.; Karp, M.; Tuukkanen, S. Engineering and Characterization of Bacterial Nanocellulose Films as Low Cost and Flexible Sensor Material. ACS Appl. Mater. Interfaces 2017, 9, 19048-19056.

14. Jeon, J.H.; Oh, I.K.; Kee, C.D.; Kim, S.J. Bacterial Cellulose actuator with electrically driven bending deformation in hydrated condition. Sens. Actuators B: Chem. 2010, 146, 307-313.

15. Di Pasquale,G.; Graziani, S.; Pollicino, A.; Trigona, C. "Paper" Based Sensor for Deformation Measurements, IEEE-I2MTC2019, in press.

16. Di Pasquale, G.; Graziani, S.; Pollicino, A.; Trigona, C. Green Inertial Sensors based on Bacterial Cellulose. In proceedings of 2019 IEEE Sensors Applications Symposium (SAS), Sophia Antipolis, France, 11-13 March 2019; pp. 1-4.

17. Pourreza, N.; Golmohammadi, H.; Naghdi, T.; Yousefi H. Green in-situ synthesized silver nanoparticles embedded in bacterial cellulose nanopaper as a bionanocompositeplasmonicsensor. Biosens. Bioelectron. 2015, 74, 353-359.

(C) 2019 by the authors. Licensee MDPI, Basel, Switzerland. This article is an open access article distributed under the terms and conditions of the Creative Commons Attribution (CC BY) license (http://creativecommons.org/licenses/by/4.0/). 